\title{
Expression of stathmin in human uterus and decidualizing endometrial stromal cells
}

\author{
Kazuhiro Tamura, Mikihiro Yoshie, Hirotaka Nishi ${ }^{1}$, Yumi Osakabe ${ }^{1}$, Keiichi Isaka ${ }^{1}$, \\ Takahiko Hara² and Hiroshi Kogo
}

Department of Endocrine Pharmacology, Tokyo University of Pharmacy and Life Science, 1432-1, Horinouch, Hachioji, Tokyo 192-0392, Japan, ${ }^{1}$ Department of Obstetrics and Gynecology, Tokyo Medical University, 6-7-1 Nishishinjuku, Shinjuku-ku, Tokyo 160-0023, Japan and ${ }^{2}$ Stem Cell Project Group, The Tokyo Metropolitan Institute of Medical Science, 3-18-22 Honkomagome, Bunkyo-ku, Tokyo 113-8613, Japan

Correspondence should be addressed to K Tamura; Email: hiro@ps.toyaku.ac.jp

\begin{abstract}
The cytosolic phosphoprotein stathmin is upregulated at the site of embryo implantation in the rodents. However, stathmin expression in the human uterus has not yet been investigated. The distribution of uterine and placental stathmin was analyzed by immunohistochemistry, while stathmin mRNA expression was detected in endometrial tissues by the reverse transcriptase-PCR. Cultured endometrial stromal cells were used to investigate whether stathmin plays a role in decidualization. Stathmin is expressed specifically in the glandular epithelium and the stromal cells of human endometrial tissue. It is also expressed by cytotrophoblasts and extravillous trophoblasts, but not by syncytiotrophoblasts or decidual tissues during the first trimester of pregnancy. When stromal cells isolated from normal endometrial tissues were cultured and stimulated to decidualize by progesterone (P4) plus estrogen or dibutyryl cyclic 3',5'-AMP, their total and phosphorylated stathmin levels decreased. Knocking down stathmin expression in the cultured stromal cells using small interfering RNA, before the cells were exposed to the decidualizing agents, significantly suppressed decidualization, as indicated by the decreased expression of IGF-binding protein-1 and prolactin. Stathmin is differently expressed in human endometrial and placental cells and may participate in the decidualization of endometrial stromal cells.

Reproduction (2006) 132 625-636
\end{abstract}

\section{Introduction}

Stathmin, also referred to as Op18 (Hailat et al. 1990) and p19 (Pasmantier et al. 1986), is a highly conserved $19 \mathrm{kDa}$ phosphoprotein among vertebrate that is an intracellular modulator rapidly phosphorylated in response to external signals (Curmi et al. 1999, Rubin \& Atweh 2004). It is a regulator of microtubule (MT) dynamics during cell-cycle progression; specifically, in vitro and in vivo evidence suggests that it promotes MT disassembly (Belmont \& Mitchson 1996, Nogales et al. 2003). Stathmin, therefore, increases MT turnover and thereby aids the rapid reorganization of the MT cytoskeleton. It also promotes tubulin catastrophes by binding to MT plus ends, thereby stimulating the exposed $\beta$-subunit of the terminal tubulin dimers to hydrolyze GTP (Nogales et al. 2003). The extensive phosphorylation of stathmin by various kinases has been shown to turn off its MT-destabilizing activity, which in turn promotes tubulin polymerization (Gavet et al. 1998, Gradin et al. 1998).
While the majority of research on stathmin function has focused on its role in controlling MT dynamics, it is possible that stathmin may interact with cell typespecific intracellular proteins and thereby play additional roles, in particular physiological processes (Curmi et al. 1999). Supporting this notion, we observed previously that stathmin expression in rodent uteri is upregulated at the site of embryo implantation (Tamura et al. 2003). Moreover, it becomes highly expressed in the decidual zone during the decidualization process (Yoshie et al. 2004). In addition, the stathmin family genes, including SCG10, SCLIP, and RB3, have also been detected in the murine uterus during implantation and the decidualization period (Yoshie et al. 2006). While the potential role(s) of uterine stathmin in pregnancy establishment has not yet been determined; our observations suggest that stathmin may be important for the decidualization of endometrial stromal cells in rodents. It has been recently shown that stathmin mRNA is expressed widely in human tissues (Bieche et al. 2003) 
and its expression levels in reproductive tissues, such as testis, ovary, uterus, and placenta, are considerably high when compared with its expression in the nervous system (Bieche et al. 2003). Moreover, stathmin is not only expressed in these tissues as the stathmin family genes, but also expressed with a similar distribution. This suggests that the putative role of stathmin plays in the rodent uterus may also occur in the human uterus.

During the menstrual cycle in humans, the uterine endometrial stromal cells transform spontaneously into decidualized stromal cells. During the process of decidualization, endometrial stromal cells alter morphologically and begin to secrete specific decidual proteins (Tabanelli et al. 1992). In in vitro studies, stromal cell preparations decidualize when ovarian steroids (P4 and estradiol) or agents that activate the CAMP pathway are added (Popovici et al. 2000). To assess the role played by stathmin in the decidualization of human endometrium, we have determined the expression of stathmin in the human uterus during the menstrual cycle. Since adequate decidualization, along with the development of the placenta, is also an essential step in the establishment of pregnancy, we also examined stathmin expression during the first trimester of pregnancy. In addition, we examined the effect of stathmin-specific small interfering RNA (siRNA) on stathmin levels of cultured endometrial stromal cells and their in vitro decidualization.

\section{Materials and Methods}

\section{Tissue samples}

Endometrial samples were obtained from patients $(n=42)$ undergoing surgery or biopsies (e.g. hysterectomy for benign indications). The sample collection was conducted with the informed consent of the patients in accordance with the requirements of the clinical research ethics committee of the Tokyo Medical University Hospital. All patients had regular 28-32-day menstrual cycles. The period in the menstrual cycle when the endometrial samples were harvested was histologically evaluated according to the standard criteria (Noyes et al. 1950) by an experienced histologist at the Department of Pathology. These data were used along with the patients' menstrual history and serum hormonal levels to establish when the samples were collected during the menstrual cycle. As a result, it was determined that 7 samples were in the menstrual phase (days 1-5), 13 in the proliferative phase (days 6-18), 12 in the early or mid-secretory phase (days 19-24), and 10 in the late secretory phase (days 25-32). Tissue samples were finely minced with scissors, washed in PBS, snap-frozen, and stored at $-80^{\circ} \mathrm{C}$ until RNA extraction. Placental samples were also obtained with informed consent from three women undergoing dilatation and curettage. A portion of endometrial and placental samples was immediately fixed in $4 \%$ paraformaldehyde dissolved in PBS, dehydrated, and embedded in paraffin; the remaining samples were stored at $-80{ }^{\circ} \mathrm{C}$ for RNA extraction. Sections $(4 \mu \mathrm{m})$ were placed on poly-L-lysine-coated glass slides (Matunami, Tokyo, Japan). Three sections at each stage were analyzed for immunostaining of stathmin.

\section{Immunohistochemistry}

Immunostaining of stathmin was performed using an antistathmin serum, which was kindly provided by Dr A Sobel of the Institut du Fer a Moulin, Paris, France and has been described previously (Tamura et al. 2003). This antiserum was raised in rabbits and cross-reacts with human stathmin. After incubation with anti-stathmin serum $(1: 100)$ in PBS containing $2 \%(\mathrm{v} / \mathrm{v})$ normal goat serum, and then with a biotinylated anti-rabbit secondary antibody, a peroxidase-conjugated streptavidin complex (VECTASTAIN ABC kit, Vector Lab., Inc., Burlingame, CA, USA) was applied. Antibody diluent that included normal rabbit serum $(1: 100)$ served as a negative control. Signals were visualized with 3,3'-diaminobenzidine and the samples were counterstained with methyl green, and cytotrophoblasts and decidual cells were stained with anti-cytokeratin antibody (Progen Biotechnik GMBH, Heidelberg, Germany) and anti-desmin antibody (Clone D33, DAKO, Glostrup, Denmark) respectively. With regard to the intensity and distribution of stathmin staining in the endometrium, the immunohistochemical localization was scored in a semi-quantitative fashion, as described previously (Budwit-Novotny et al. 1986). These evaluations involved the glandular epithelium and stromal cells only, as these cells showed the most staining. All evaluations were performed independently by two examiners and the average and $\mathrm{H}$-score values ( $n=4$ or 5 ) were then calculated. These values were used to compare the stathmin levels in both the cell types between the functional zone and the stratum basale of the endometrium.

\section{$R N A$ extraction and real-time reverse transcriptase (RT)-PCR}

Total RNA was isolated using the Isogen reagent (Nippon Gene, Tokyo, Japan) and quantified by A260/A280 measurement using an Ultraspec 3000 (Amersham Biosciences Corp.). Five micrograms of total RNA were reverse-transcribed into cDNA using an RT-PCR kit (Stratagene, La Jolla, CA, USA) according to the manufacturer's recommendations. Real-time PCR was then performed to quantitate the specific RNA levels. Thus, $20 \mu \mathrm{l}$ PCRs were set up with final concentrations of $5 \mathrm{mM} \mathrm{MgCl} 2,2 \mu \mathrm{l}$ SYBR green master mix (Roche Diagnostics), $5 \mu$ of $1: 10$ diluted cDNA, and $0.3 \mu \mathrm{M}$ of both forward and reverse primers. Stathmin was 
amplified using the following primer pair: $5^{\prime}$ TTCTCCCCATCCCCTTCCTA-3' (forward), 5'-GCCATTAACCCAGTACACCAAG-3' (reverse). The reactions were then cycled in the LightCycler (Roche Diagnostics) with the following parameters: denaturation for one cycle at $95{ }^{\circ} \mathrm{C}$ for $10 \mathrm{~s}$ followed by 40 cycles at $95^{\circ} \mathrm{C}$ for $10 \mathrm{~s}, 60^{\circ} \mathrm{C}$ for $10 \mathrm{~s}$, and $72{ }^{\circ} \mathrm{C}$ for $8 \mathrm{~s}$ with a temperature transition rate of $20^{\circ} \mathrm{C} / \mathrm{s}$. Fluorescence was read at $72{ }^{\circ} \mathrm{C}$ and the melting curve employed continuous fluorescence reading (ABI PRISM 7000, Applied Biosystem Japan Ltd, Tokyo, Japan). The LightCycler software generated a standard curve (measurements taken during the exponential phase of the amplification) that enabled the quantification of gene expression in each test sample. The stathmin mRNA level was normalized to the glyceraldehyde-3-phosphate dehydrogenase (G3PDH) mRNA levels. The individual raw data or the mean \pm S.E.M. are shown. Statistical significance was determined using Student's $t$-test and ANOVA.

\section{Endometrial cell preparation and experimental schedule}

Endometrial stromal cells were isolated from normal endometrial tissue samples using the procedures of Satyaswaroop et al. (1979) with slight modifications. The stromal cells were then resuspended in basal medium (Dulbecco's modified Eagle's medium (DMEM)/F12 without phenol red; Life Technologies) containing $50 \mathrm{U} / \mathrm{ml}$ penicillin, $50 \mu \mathrm{g} / \mathrm{ml}$ streptomycin, and $0.25 \mu \mathrm{g} / \mathrm{ml}$ fungizone (Life Technologies) along with $10 \%(\mathrm{v} / \mathrm{v})$ charcoal/dextran-treated fetal bovine serum (10\% stripped fetal bovine serum (FBS) HyClone, South Logan, UT, USA), and seeded in a tissue culture flask (Asahi Techno Glass, Tokyo, Japan; $25 \mathrm{~mm}^{2}$ ). The medium was removed $2 \mathrm{~h}$ later and the adherent cells were washed with fresh medium and cultured to subconfluency for 7 days. Finally, the cells were passaged into 12-well culture plates with basal medium containing $10 \%$ stripped FBS and antibiotics and cultured until confluency. As shown in Fig. 1, to induce decidualization, the cells were cultured for 3-15 days in the basal medium ( $1 \mathrm{ml} /$ well) containing $2 \%$ stripped FBS and antibiotics supplemented with (A) $1 \mu \mathrm{M}$ P4 plus $10 \mathrm{nM} 17 \beta$-estradiol (E2) or (B) $1 \mathrm{mM}$ dibutyryl cAMP (db-cAMP; Popovici et al. 2000). The medium was changed every 2 or 3 days in the steroid-treated decidualization model and every day in the db-cAMPinduced decidualization model. The culture media were examined for IGF-binding protein-1 (IGFBP-1) and prolactin (PRL) levels, which are well-known decidual markers, while the cultured cells were lysed. The lysates were subjected to immunoblotting analysis or the $\operatorname{poly}(\mathrm{A})^{+}$RNA was extracted using the QuickPrep microRNA Purification Kit (Amersham) and used in semi-quantitative RT-PCR analysis. To knock down stathmin expression, the stromal cells were pretreated

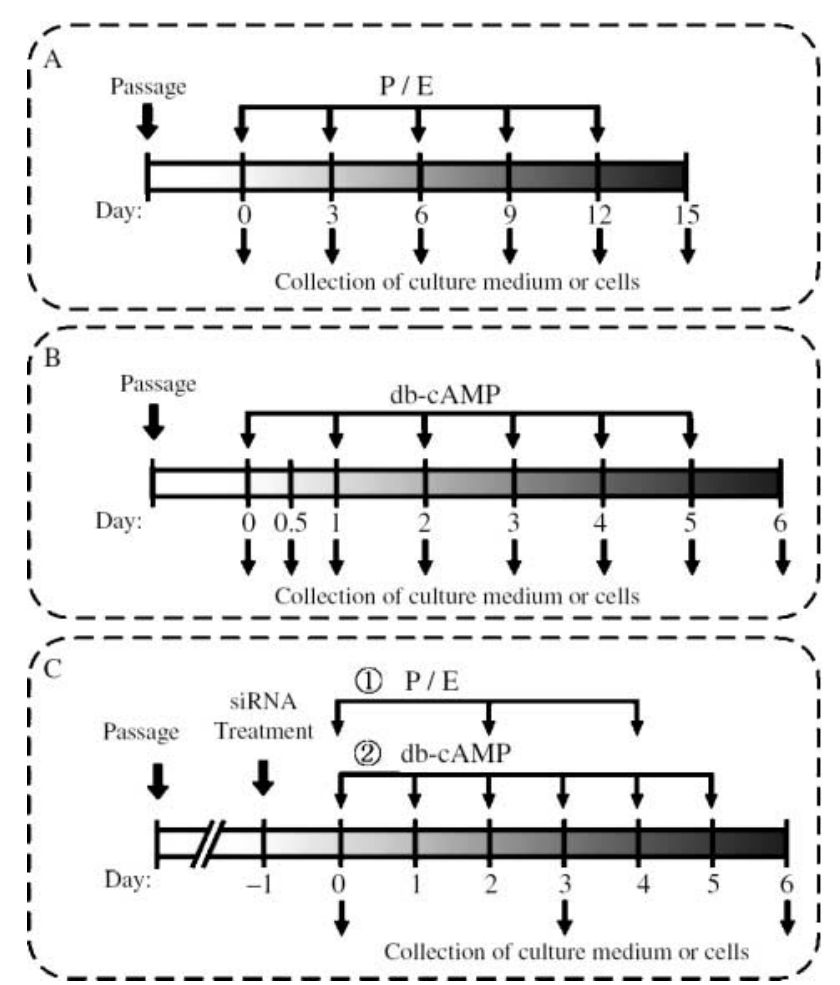

Figure 1 Schematic depiction of the experimental schedule used to induce the decidualization of cultured endometrial stromal cells. Decidualization was induced by treating the cells with (A) either $1 \mu \mathrm{M}$ progesterone (P4) plus $10 \mathrm{nM} 17 \beta$-estradiol (E2) (P/E) (B) or $1 \mathrm{mM}$ dibutyryl cAMP (db-cAMP). The medium was changed every 2 or 3 days in the steroid-treated decidualization model and every day in the db-cAMP-induced decidualization model. The IGF-binding protein-1 (IGFBP-1) and prolactin (PRL) levels in the culture media were then assessed while the cultured cells were lysed. The lysates were subjected to immunoblotting analysis or their poly $(\mathrm{A})^{+}$RNA was extracted and subjected to RT-PCR. In the experiment, where stathmin expression was knocked down $(\mathrm{C})$, the cells were pretreated with stathmin siRNA for $24 \mathrm{~h}$ before being stimulated to decidualize.

with the stathmin siRNA (see below for details) for $24 \mathrm{~h}$ before stimulating decidualization, as shown in Fig. 1C.

\section{Semi-quantitative RT-PCR}

$\operatorname{Poly}(\mathrm{A})^{+}$RNA was reverse-transcribed using a One Step RNA Kit (TaKaRa, Siga, Japan) and PCR amplification was performed as described previously (Tamura et al. 2000). Oligonucleotide primers (5'-GTCGCTTGTCTTCTATTCACC- $3^{\prime}$ and 5'-ATGGGGAGAAAGTCAGTTCTC-3') specific for human stathmin that produced a 500 bp PCR-amplified product were used. RT-PCR was also performed with primers specific for IGFBP-1 and PRL transcripts; these primers have been described by Brosens et al. (2000) and the amplification protocol for both products was the same as that for stathmin. As an internal control, specific G3PDH primers (BD Biosciences, PaloAlto, CA, USA) were used. 


\section{Western blot analysis}

The harvested culture medium was stored at $-30{ }^{\circ} \mathrm{C}$ until analysis. Cells were lysed by adding Chaps Cell Extract Buffer (Cell Signaling Technology, Inc., Beverly, MA, USA) and the lysates were prepared for immunoblotting according to the manufacturer's instructions. The culture media $(15 \mu \mathrm{l})$ and the lysate samples $(3 \mu \mathrm{g}$ protein) were subjected to $15-25 \%$ gradient SDS-PAGE (Daiichi Pure Chemicals Co., Ltd, Tokyo, Japan) and then electrotransferred onto PVDF membranes (Millipore, Bedford, MA, USA). The membranes were incubated with rabbit anti-human PRL antibody (1:1000; SAMP \#518, anti-hPRL-IC-5, National Hormone and Peptide Program), rabbit anti-human IGFBP-1 antiserum (1:1000; Upstate Biotechnology, Lake Placid, NY, USA), or the anti-stathmin serum (1:10 000), which can specifically detect the single $19 \mathrm{kDa}$ band in even crude cell lysates of endometrial stromal cells (data not shown). For the detection of phosphorylated stathmin, we used a native PAGE system that separates stathmin phosphoisomers according to the charge differences introduced by each of the four identified phosphorylation events (Gavet et al. 1998, Gradin et al. 1998). The blot was incubated with anti-phosphorylated stathmin antibody (1:100; p-Op18: Ser16, Santa Cruz Biotechnology, Inc., Santa Cruz, CA, USA). This antibody, which was raised against a peptide corresponding to a short amino acid sequence that included phosphorylated Ser16 of human stathmin, does not recognize nonphosphorylated stathmin. After the detection of phosphorylated stathmin, the same membrane was incubated in stripping solution $(75.3 \mathrm{mM}$ Tris- $\mathrm{HCl}(\mathrm{pH} 6.7)$ containing $2 \% \quad(\mathrm{w} / \mathrm{v}) \quad$ SDS and $0.007 \% \quad(\mathrm{v} / \mathrm{v})$ $\beta$-mercaptoethanol) and reprobed for PRL or IGFBP-1. The expression of $\beta$-actin was examined using a mouse monoclonal anti- $\beta$-actin antibody (1:10 000; SigmaAldrich) as a loading control. Goat anti-mouse or antirabbit IgG (Vector Lab., Inc.; $0.5 \mu \mathrm{g} / \mathrm{ml}$ ) conjugated with horseradish peroxidase served as the secondary antibody for each analysis. All blotting experiments were repeated at least twice and the representative data are shown.

\section{SiRNA treatment}

The siRNAs used were purchased from Santa Cruz Biotechnology, Inc. (the Op18 siRNA is sc-36127, while the irrelevant control siRNA is sc-37007) and transfected into stromal cells according to the manufacturer's protocol. Stromal cells were treated for $24 \mathrm{~h}$ with either of the two siRNAs. The transfection specifically inhibited stathmin mRNA within $24 \mathrm{~h}$, leading to the downregulation of its protein, as measured by western blot analysis (see Fig. 7). After $24 \mathrm{~h}$ siRNA treatment, the cells were stimulated with $1 \mu \mathrm{M} \mathrm{P} 4$ and $10 \mathrm{nM}$ E2 or $1 \mathrm{mM}$ db-cAMP (Fig. 1C).

\section{Proliferation assay}

To determine the effect of knocking down stathmin on the capacity of endometrial cells to proliferate, changes in cell numbers after stathmin siRNA treatment were monitored by a proliferation bioassay. Thus, endometrial stromal cells $\left(4 \times 10^{4}\right)$ were seeded in a 24-well culture plate in DMEM/F12 medium containing $10 \%$ FBS and cultured for $24 \mathrm{~h}$ before siRNA transfection. At 24- and $48 \mathrm{~h}$ culture after siRNA treatment, the cells were incubated with WST-1 reagent (Cell Counting Kit, Dojindo, Tokyo, Japan) for $15 \mathrm{~min}$. The media were then collected and the staining intensity was measured spectrophotometrically with a microtiter plate reader. The data were expressed as ratios of the control value.

\section{Results}

\section{Uterine stathmin mRNA levels during the menstrual cycle}

To characterize the expression of stathmin in human endometrium during the menstrual cycle, 42 samples of human endometrial tissue harvested at varying defined phases of the endometrial cycle were subjected to realtime RT-PCR analysis (Fig. 2). Stathmin mRNA levels were high in the proliferative phase and tended to decrease in the late secretory phase, but overall there were no statistical differences in the stathmin mRNA levels at different phases of the menstrual cycle. Thus, significant changes in the mRNA expression of stathmin in human endometrial tissue during the menstrual cycle were not observed.

\section{Localization of stathmin protein in the uterus during the menstrual cycle}

We then studied the distribution of stathmin in the human uterus during the menstrual cycle by subjecting three of the human endometrial tissues harvested on days 13, 18, and 24 of the menstrual cycle to immunohistochemical staining (Fig. 3). The specificity of immunoreactivity was confirmed by a negative control in which the primary antibody was omitted (Fig. 3F). In general, signals were detected in the glandular epithelium and stromal cells, but the intensity of staining was stronger in the glandular epithelium than in the stroma cells (Fig. 3A-D). Stathmin signals were also found in blood vessels (Fig. 3D). Stathmin immunostaining in the myometrium was faint as seen in Fig. 3A. Marked changes in stathmin protein levels in human endometrial tissue during the menstrual cycle were not observed. When we subjected the stained tissues to $\mathrm{H}$-score analysis (Table 1 ), we found that the immunoreactivity was significantly stronger in stromal cells in the functional zone, especially those near the uterine lumen, than in those in the stratum basale 


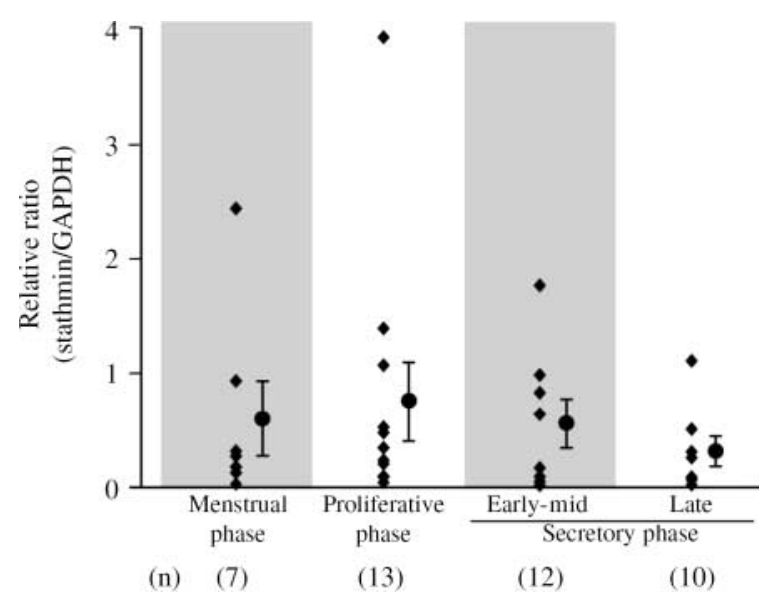

Figure 2 Expression of stathmin mRNA in human endometrial tissues collected at different phases of the menstrual cycle. Endometrial tissues from 42 patients with regular menstrual cycles were removed, frozen, stored at $-80^{\circ} \mathrm{C}$, and their total extracted RNA was subjected to realtime RT-PCR analysis. The endometrial tissues were determined to be in particular phases of the endometrial cycle as described in Materials and Methods, i.e. either in the menstrual phase (days 1-5), the proliferative phase (days 6-18), and the early or mid-secretory phase (days 19-24), or in the late secretory phase (days 25-32). The values obtained were normalized against G3PDH mRNA levels. The squares show individual raw value, and the dots with bars in each menstrual phase show the mean \pm S.E.M.

residing in the endometrium near the myometrium; this was true for endometrial tissues collected on days 13, 18, and 24 (Table 1 and data not shown). However, the intensity of staining did not differ between the various days of the menstrual cycle that we tested (data not shown). Thus, neither the protein nor the mRNA expression of stathmin appears to change during the menstrual cycle.

\section{Localization of stathmin protein in the first-trimester placenta}

Our observations described previously confirmed that stathmin protein is expressed in the human uterus during the menstrual cycle. Consequently, we examined its expression in the pregnant human uterus by studying samples of the first-trimester placenta (Fig. 4). Strong stathmin immunostaining was observed in cytotrophoblast cells, but not in syncytiotrophoblast cells. The extravillous cytotrophoblast, which is confirmed by the immunostaining of cytokeratin, also exhibited intense signals (Fig. 4B), whereas decidual tissue stained weakly (Fig. 4F). Interestingly, vascular smooth muscle in decidua had intense signals for desmin, a decidual marker (Fig. 4G). No staining was observed in serial sections that had been stained with the secondary antibody in the absence of primary antibody (Fig. 4D and $\mathrm{H}$ ). Thus, stathmin is also expressed in particular cells of the first-trimester human placenta.

\section{Stathmin expression in cultured endometrial stromal cells undergoing steroid-induced decidualization}

Human endometrial stromal cells decidualize in vitro in response to a combination of P4 and E2 (Irwin et al. 1989, Kasahara et al. 2001). Thus, to examine whether stathmin expression in endometrial stromal cells changes during decidualization, we employed this in vitro decidualization model. To this end, we isolated the endometrial stromal cells from normal endometrial tissue samples and cultured them as described in 'Materials and Methods'. Decidualization was then induced as shown in Fig. 1 by adding P4 and E2. First, we confirmed the decidualization of the cultured endometrial stromal cells by examining whether they secrete enhanced levels of PRL and IGFBP-1 and exhibit morphological changes that are markers of decidualization. Western blot analysis of the conditioned media showed that while the control cells cultured without the steroids produced very little PRL and IGFBP-1, the addition of P4 and E2 to the medium markedly increased endometrial stromal cell secretion of both markers over 6 days culture (Fig. 5A). With regard to the morphology of the endometrial stromal cells, while the control cells maintained a fibroblastic appearance until day 15 , the cells exposed to P4 and E2 became enlarged and had indistinct borders (data not shown). We then examined the expression of stathmin during the in vitro decidualization of the endometrial stromal cells (Fig. 5A). Stathmin protein levels tended to be lower on day 6 after steroid treatment than on day 0 of culture, however by days 9,12 , and 15 , the levels were markedly decreased (Fig. 5A and C). RT-PCR analysis revealed that stathmin mRNA expression decreased during the 6 day culture (Fig. 5B).

\section{Stathmin expression in cultured endometrial stromal cells undergoing $d b$-cAMP-induced decidualization}

It has been shown that the in vitro decidualization of endometrial stromal cells can also be initiated by treatment with a cAMP analog (Tang et al. 1993). Consequently, to confirm the effect of decidualization on stathmin expression, we examined the stathmin mRNA and protein levels in cultured endometrial stromal cells subjected to db-cAMP-induced decidualization. Figure 6 shows that db-cAMP-induced decidualization enhanced the mRNA (A) expression and (B) secretion of IGFBP-1 and PRL on days 3 and 6 of culture respectively. In contrast, stathmin mRNA levels in the db-cAMP-treated cells had decreased by days 3 or 6 (Fig. $6 \mathrm{~A}$ ), which is consistent with the results obtained with the steroid-treated decidualization model (Fig. 5B). Furthermore, western blot analysis with native PAGE and an anti-phosphorylated (Ser16) stathmin antibody showed that the levels of phosphorylated stathmin, especially the stathmin form with three or four phosphorylated sites, had also decreased in decidual cells by day 3 (Fig. 6C). This was confirmed when cell lysates were harvested at varying times between days 0 and 3 

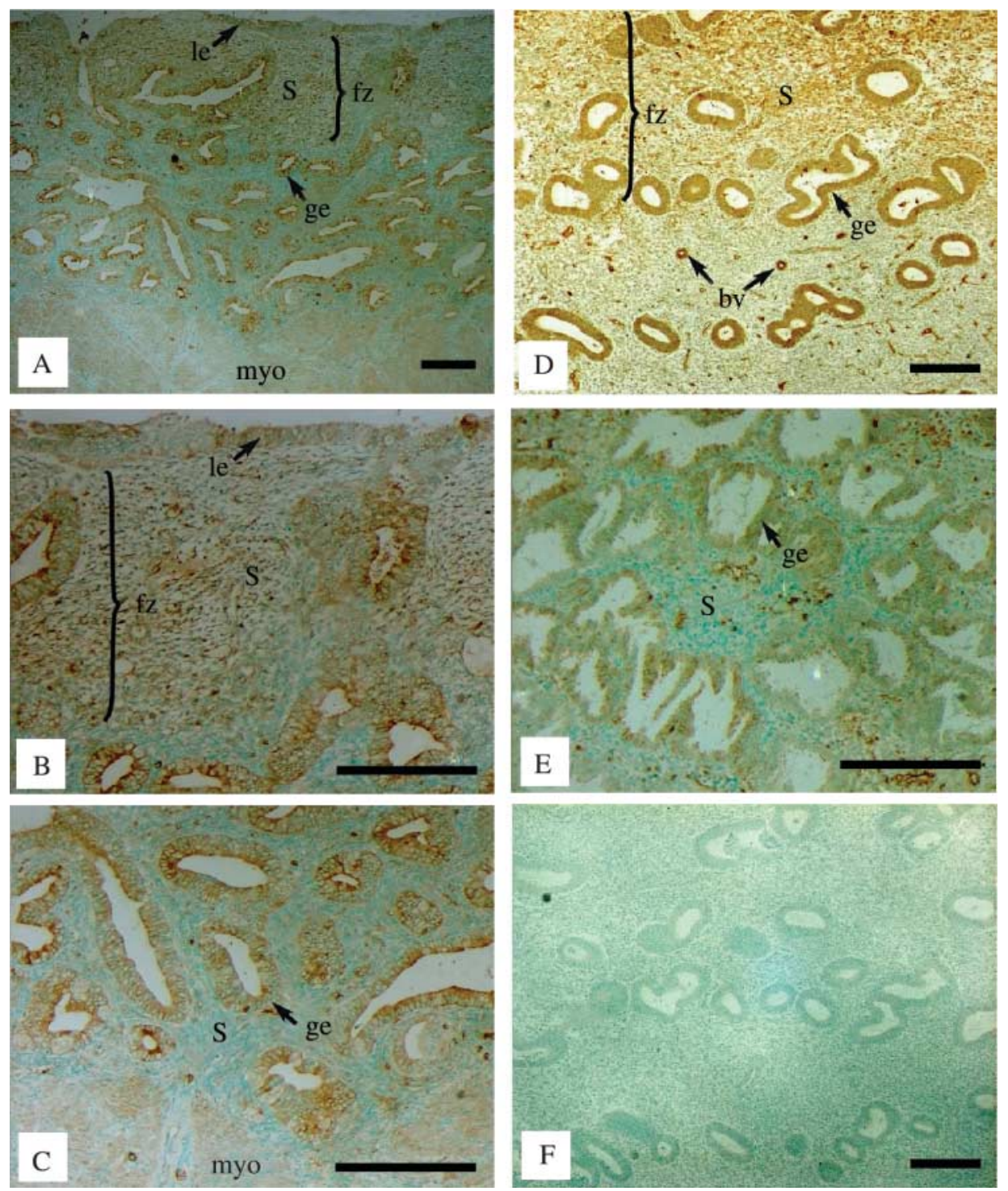

Figure 3 Expression of stathmin protein in human endometrial tissues collected at different phases of the menstrual cycle. Shown are representative of immunostained endometrial tissues harvested in (A)-(C) the proliferative phase (day 13), (D) the early secretory phase (day 18), or (E) the late secretory phase (day 24). (F) Negative control of a tissue in the early secretory phase, where only the secondary antibody was added in the procedure. Original magnification: (A) $50 \times,(B)$ and (C) 126×, (D)-(F) $63 \times$. Bars represent $200 \mu \mathrm{m}$. fz, functional zone of endometrium; le, luminal epithelium; ge, glandular epithelium; s, stroma; myo, myometrium; bv, blood vessel.

Table 1 Comparison of $\mathrm{H}$-score in stathmin levels in glandular epithelium and stromal cells in the functional area and the stratum basale of the endometrium on days 13 and 18 .

\begin{tabular}{|c|c|c|c|c|c|c|c|c|}
\hline & \multicolumn{4}{|c|}{ Day 13} & \multicolumn{4}{|c|}{ Day 18} \\
\hline & \multicolumn{2}{|c|}{ Functional } & \multicolumn{2}{|c|}{ Basale } & \multicolumn{2}{|c|}{ Functional } & \multicolumn{2}{|c|}{ Basale } \\
\hline & ge & $\mathrm{s}$ & ge & s & ge & $S$ & ge & $\mathrm{s}$ \\
\hline 0 & 0 & 3 & 0 & 57 & 0 & 6 & 0 & 92 \\
\hline Grade & & & & & & & & \\
\hline $1+$ & 29 & 41 & 40 & 61 & 55 & 15 & 36 & 16 \\
\hline $2+$ & 197 & 169 & 173 & 45 & 218 & 160 & 158 & 2 \\
\hline $3+$ & 79 & 83 & 88 & 0 & 8 & 92 & 40 & 0 \\
\hline H-score ( \pm s.E.M.) & 305 (19.4) & $295(22.1)$ & $302(24.0)$ & $167 *(34.8)$ & $281(14.6)$ & $303(7.7)$ & $301(3.4)$ & $109^{\dagger}(4.0)$ \\
\hline
\end{tabular}

The intensity of stathmin signals in immunohistochemistry (four or five pictures, including Fig. 3) was characterized as follows: not present (0), weak but detectable $(1+)$, distinct $(2+)$, and very strong $(3+)$. Each value of the $\mathrm{H}$-score in glandular epithelium (ge) and stromal (s) cells was calculated separately in the area of the functional area (functional) and of the stratum basale (basale) in the endometrium. The average scores with each grade of intensity $(0-3+)$ and the mean \pm S.E.M. of $\mathrm{H}$-score in ge and s are shown. ${ }^{*} P<0.05,{ }^{\dagger} P<0.01$; versus stromal cells in the functional area on each day. 

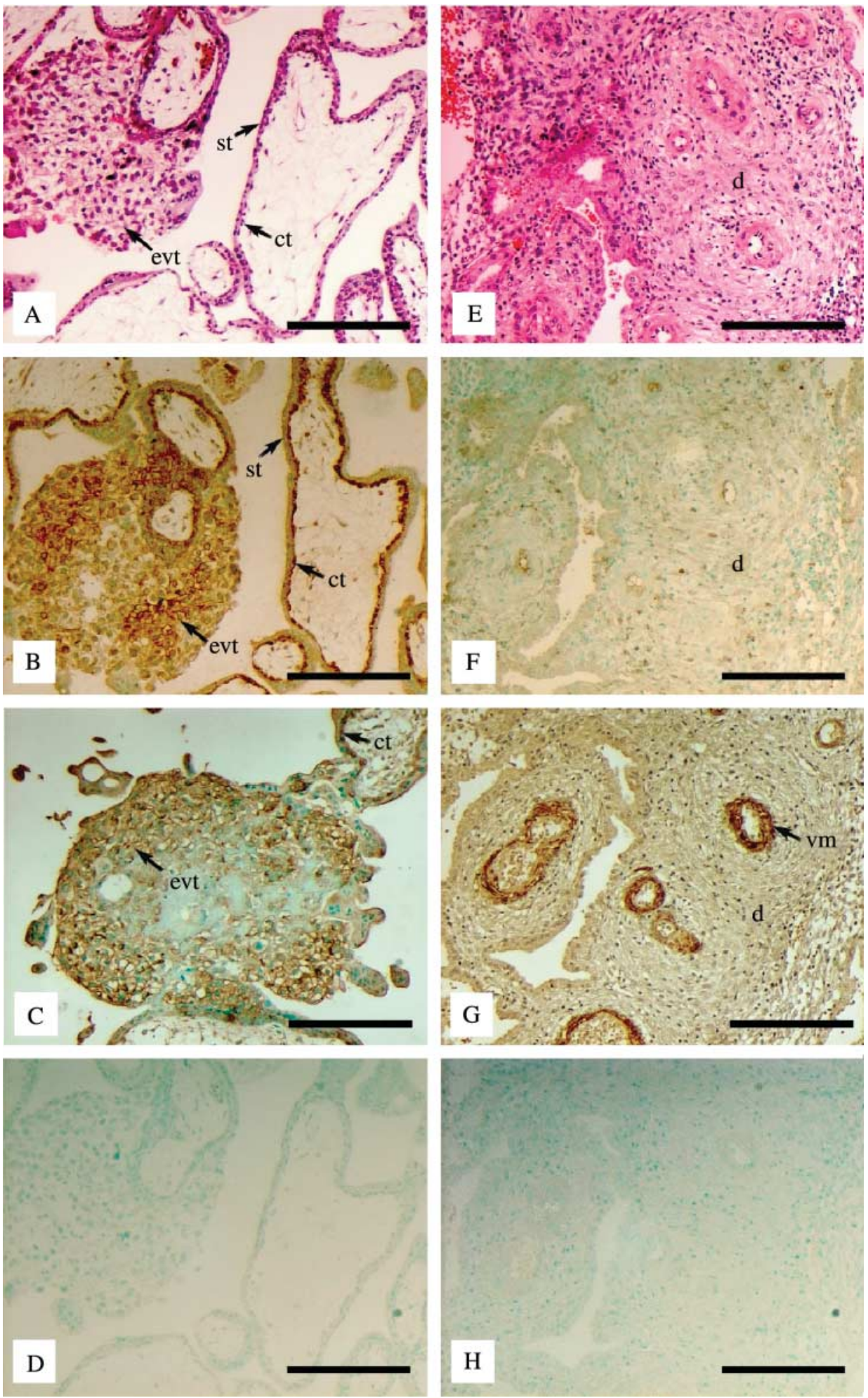

Figure 4 Expression of stathmin protein in human first-trimester placentas. Shown are examples of the chorionic villi (A)-(D) and decidual tissues (E)- $(\mathrm{H})$ of first-trimester placentas that were immunostained to determine stathmin expression $(B)$ and $(F)$, stained with hematoxylin-eosin $(A)$ and $(E)$, with cytokeratin, a marker of cytotrophoblast (C), or with desmin, a marker of decidual cells (G). (D), (H): Negative control. Original magnification: $126 \times$. Bars represent $200 \mu \mathrm{m}$. ct, cytotrophoblasts; st, syncytiotrophoblasts; evt, extravillous cytotrophoblasts; d, deciduas; vm, vascular smooth muscle.

(72 h) and subjected to the same western blotting analysis (Fig. 6D). The decrease in the phosphorylated forms of stathmin was initiated within $12 \mathrm{~h}$ db-cAMP treatment, namely, before significant increases in IGFBP-1 levels were observed in the culture media.

\section{Effect of knocking down stathmin in endometrial stromal cells on their in vitro decidualization and proliferation}

To determine whether stromal stathmin expression participates in in vitro decidualization, we analyzed 

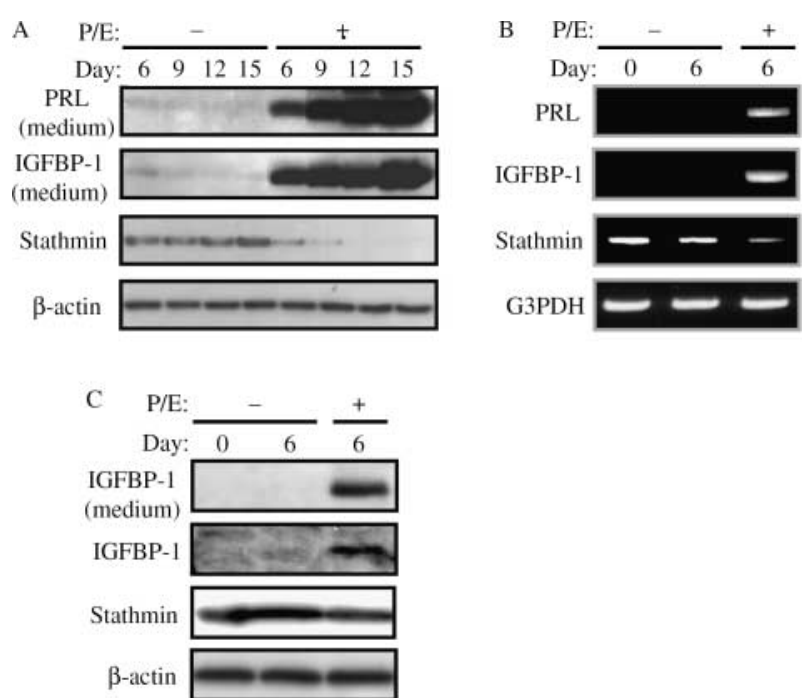

Figure 5 Stathmin expression in cultured endometrial stromal cells during their steroid-induced decidualization. Stromal cells were cultured for 15 days in stripped FBS-supplemented DMEM/F12 in the presence or absence of $1 \mu \mathrm{M} \mathrm{P} 4$ and $10 \mathrm{nM} \mathrm{E} 2$ (P/E). Culture media were collected and changed every 2-3 days. Day 0 is prior to the start of culture, at which point the cells had not been exposed to P/E. (A) Kinetics of the production of IGFBP-1, PRL, and the levels of stathmin. The conditioned media and cell lysates were subjected to western blot analysis on days $0-15$. The numbers at the top of the figures indicate the day of culture. (B) Expression of IGFBP-1, PRL, and stathmin mRNAs on days 0 and 6 of culture. The poly $(A)^{+}$RNA extracted from the cells on day 6 of culture was subjected to semi-quantitative RT-PCR. G3PDH served as an internal control. (C) Expression of IGFBP-1 and stathmin protein. Lysates of cells harvested each day were subjected to western blot analysis ( $3 \mu \mathrm{g}$ protein per lysate). The levels of IGFBP- 1 protein in the day 6 culture media were also analyzed (top panel).

the effect of knocking down stathmin mRNA expression in cultured endometrial stromal cells before they were induced to decidualize (Fig. 7). As expected, when the cells were treated with stathmin siRNA before being exposed to db-cAMP, a marked decrease in stathmin mRNA expression was observed (Fig. 7A, siRNA); this inhibitory effect lasted until at least day 6 . We then examined the effect of knocking down stathmin mRNA expression on endometrial stromal cell decidualization using the db-cAMP-induced decidualization model. The siRNA treatment markedly inhibited IGFBP-1 and PRL mRNA expression (Fig. 7A) and the secretion of both decidualization markers into the culture medium (Fig. 7C). The cellular stathmin and IGFBP-1 levels were also markedly inhibited by the siRNA treatment (Fig. 7B). Inhibition of IGFBP-1 expression by stathmin siRNA was also observed in the steroid-induced decidualization model (Fig. 7D).

We further examined the effect of knocking down stathmin expression on the proliferation rate of endometrial stromal cells that have not been stimulated to undergo decidualization. The stathmin protein levels in the siRNA-treated cells were reduced within $48 \mathrm{~h}$ (Fig. 8A). At this timepoint, cell proliferation decreased
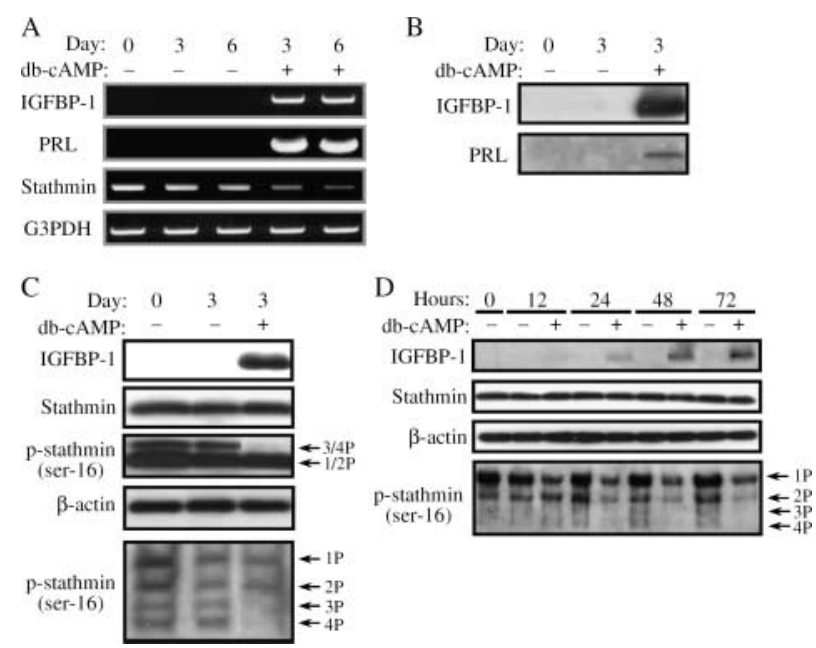

Figure 6 Stathmin expression in cultured endometrial stromal cells during their db-cAMP-induced decidualization. Stromal cells were cultured for 6 days in DMEM/F12 supplemented with 2\% FBS in the presence or absence of db-cAMP (1 mM). Culture media were collected and changed every day. (A) Expression of IGFBP-1, PRL, and stathmin mRNAs. Poly(A) ${ }^{+}$RNA was extracted from the days $0,3,6$ cells and subjected to semi-quantitative RT-PCR. G3PDH served as an internal control. (B) Levels of IGFBP-1 and PRL in the culture media harvested on day 3. Equivalent quantities of medium $(15 \mu \mathrm{l})$ were subjected to western blotting. (C) and (D) Levels of IGFBP-1, stathmin, and phosphorylated stathmin for 3 days (C) or for 0.5-3 days (D) after db-cAMP stimulation. Cell lysates containing $3 \mu \mathrm{g}$ protein were analyzed by western blot analysis. To detect phosphorylated stathmin (bottom panels of $C$ and D), the samples were subjected to western blot analysis using native PAGE and anti-phosphorylated (Ser16) stathmin antibody.

as the proliferation rate was at $80 \%$; in contrast, the stathmin protein levels and the proliferation of the control and stathmin siRNA-treated cells were equivalent at $24 \mathrm{~h}$ (Fig. 8B).

\section{Discussion}

In the present study, our results suggest that stathmin may be an intracellular regulatory factor associated with the proliferation and/or differentiation of endometrial cells during early pregnancy in humans. It is expressed specifically in the glandular epithelium and stromal cells of human endometrial tissue. It is also expressed by cytotrophoblasts and extravillous trophoblasts, but not by syncytiotrophoblasts or decidual tissues during the first trimester of pregnancy. When stromal cells isolated from normal endometrial tissues were stimulated to decidualize by $\mathrm{P} 4$ plus $\mathrm{E} 2$ or db-cAMP, their phosphorylated stathmin levels clearly decreased. However, knocking down stathmin expression in the cultured stromal cells using siRNA before the cells were exposed to the decidualizing agents significantly suppressed decidualization. Therefore, stathmin is differently expressed in human uterus and placenta, and may be involved in the decidualization of endometrial stromal cells. 

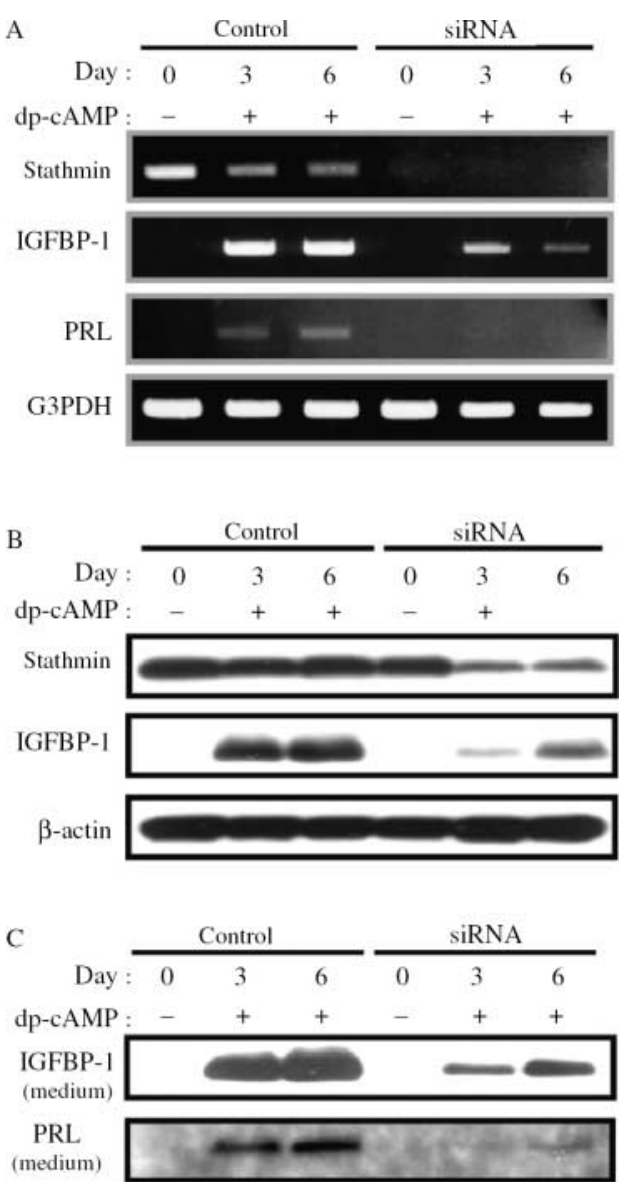

D

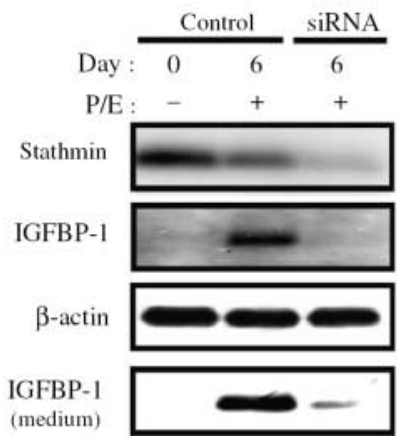

Figure 7 Effect of stathmin knockdown on the decidualization of cultured endometrial stromal cells. Stromal cells were transfected for $24 \mathrm{~h}$ with stathmin siRNA or an irrelevant control siRNA, after which the culture medium was changed to DMEM/F12 supplemented with db-cAMP (A)-(C) or $1 \mu \mathrm{M} \mathrm{P4}$ and $10 \mathrm{nM} \mathrm{E2} \mathrm{(P/E)} \mathrm{(D)} \mathrm{and} \mathrm{the} \mathrm{cells} \mathrm{were}$ cultured for an additional 6 days. (A) Confirmation of the knockdown of stathmin expression (top panel) and assessment of the effect of siRNA treatment on the IGFBP-1 and PRL mRNA levels (two middle panels). Poly $(A)^{+}$RNA was extracted and subjected to semi-quantitative RT-PCR. Effect of siRNA treatment on (B) cellular levels of stathmin and IGFBP-1 protein and (C) secreted levels of IGFBP-1 and PRL. Cell lysates $(3 \mu \mathrm{g})(\mathrm{B})$ and culture media $(15 \mu \mathrm{l})(\mathrm{C})$ harvested on days 0,3 , and 6 were subjected to western blot analysis. (D) Effect of stathmin knockdown on P/E-induced decidualization in stromal cells. Stathmin siRNA was transfected to stromal cells 1 day before P/E stimulation. The cell lysate $(3 \mu \mathrm{g})$ and culture media $(15 \mu \mathrm{l})$ were subjected to western blot analysis for stathmin and IGFBP-1.

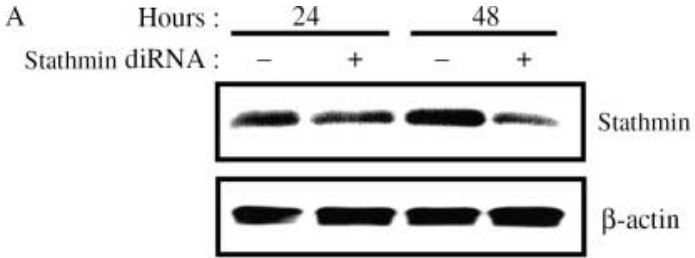

B

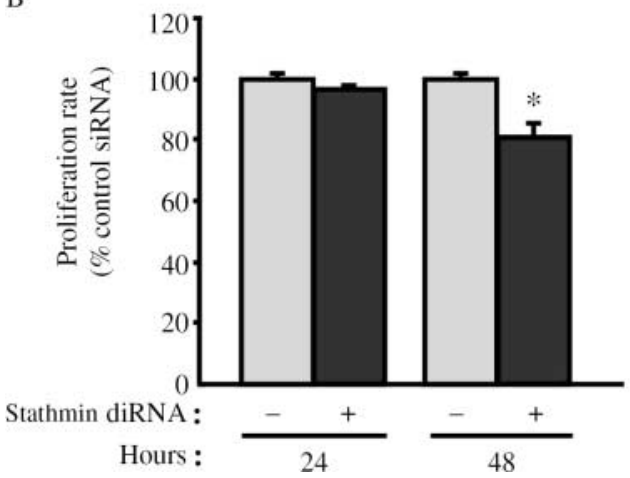

Figure 8 Effect of stathmin knockdown on the proliferation of cultured endometrial stromal cells. Stromal cells were transfected with stathmin siRNA or an irrelevant control siRNA (stathmin siRNA $(-)$ ) and cultured for 24 and $48 \mathrm{~h}$, after which the proliferation rate of the cells was assayed by using the WST-1 reagent (B). The data are represented as ratios of the control; shown are the mean \pm s.E.M. from three independent experiments. ${ }^{*} P<0.01$ vs. stathmin siRNA $(-)$. Therefore, the stathmin siRNA knocked down stathmin mRNA expression was also confirmed by western blot analysis (A).

While previous studies have shown that stathmin is prominently expressed in the nervous system and its role in the regulation of MT dynamics is highly conserved across species (Ozon et al. 1997, Curmi et al. 1999); it has not been shown previously whether the uterine stathmin expression found in rats occurs in all mammals. It has been revealed that ovarian cancer is associated with elevated stathmin expression (Price et al. 2000), but there are no previous reports that suggest stathmin participates in normal human reproductive functions. This study showed for the first time stathmin expression in human uterine endometrial and placental tissues. Within the uterine endometrium, stathmin was largely expressed in the glandular epithelium and stromal cells. Similarly, in rats, in the early stage of pregnancy, stathmin is expressed in the glandular epithelium of the uterus (Tamura et al. 2003). Although we do not have any data that would allow us to discuss the significance of stathmin expression in glandular epithelium, it is well known that leukemia inhibitory factor, which is secreted from the glandular epithelium, exerts its action through its effect on the luminal epithelium and stromal decidualization. Therefore, the function of glandular epithelium is important for the establishment of pregnancy. Since stathmin localization has been reported in a number of secretory glands, including salivary gland (Nakashima et al. 2006), mammary gland 
(Puissant et al. 1995), pheochromocytoma cells (Takekoshi et al. 1998), and the gonadotrope (Drouva et al. 1995); it might be associated with glandular proliferation or morphological changes. Interestingly, we found in our present study that the stathmin signals in human endometrial tissue were distinctly localized to the stromal cells of the functional area rather than the endometrium close to the myometrium (the stratum basale). This difference in staining intensity between the functional layer and the stratum basale of the endometrium may reflect the differences in the stromal cell populations in the endometrium. Supporting this notion, the functional area is subject to dramatic morphological changes during the proliferative, secretory, and menstrual phases that are accompanied by the proliferation and death of various cell types; it has been shown that stathmin is generally expressed at higher levels in proliferating cells than in non-proliferative cells (Rowlands et al. 1995). These observations together with our own, thus suggest that stathmin may play a physiological role in the functional area of the uterus. Supporting this notion, a previous study that implicates stathmin in decidualization in rodents and found higher levels of stathmin mRNA in human reproductive tracts than in many other tissues (Bieche et al. 2003).

Stathmin is one of the several genes, whose expression is specifically upregulated during the initiation of implantation and decidualization in rodents (Tamura et al. 2003, Yoshie et al. 2004). It's expression increased with decidualization and decreased when decidualization is terminated in rodents, whereas in human, a significant increase was not observed with the initiation of decidualization. The difference might be due to species difference. We also observed that stathmin mRNA levels tended to decrease as the endometrial tissues progressed from the proliferation phase to the late stage of the secretory phase. However, this tendency did not reach statistical significance. During the mid- to late secretory phase, stromal cells differentiate to form decidual cells, shows the enlarged and rounded cell types, and produce a variety of cytokines, which probably enhance decidualization and regulate trophoblast invasion. Further, to test the possibility that stathmin participates in decidualization, we cultured the stromal cells isolated from normal human endometrial tissue and induced them to decidualize by exposing them to either E2 plus P4 or db-cAMP. Analysis of both in vitro decidualization models revealed that when endogenous stathmin mRNA expression was knocked down by siRNA transfection prior to receiving the decidualizing stimulus, significantly lower levels of the decidualization markers IGFBP-1 and PRL were produced by the cultured stromal cells. Antisense depletion of stathmin prevents nerve growth factor-stimulated differentiation of PC12 cells into sympathetic-like neurons (Di Paolo et al. 1996). This strongly suggests that stathmin expression is likely to be involved in the preparation or initiation of decidualization. It's expression is high in undifferentiated cells and decreases markedly during the induction of differentiation in a variety of cell types (Schubart et al. 1992). High stathmin levels are also characteristic of migratory progenitors. However, as the cells begin to differentiate, its levels decrease to allow the MT network to form properly. Increased stathmin in newly formed or in preexisting oligodendrocytes in both cases results in MT instability and improper function of the cytoskeletal apparatus. It has been proposed that stathmin may help limit cell 'overproliferation' during the cell-cycle period preceding cell commitment and/or differentiation (Curmi et al. 1999). Consequently, stathmin expression in endometrial stromal cells may be crucial for determining their commitment or fate upon or prior to receive the decidualizing stimulus. Notably, we found that knocking down stathmin expression also significantly decreased stromal cell proliferation. It may exert a regulatory action on physiological stromal cell proliferation prior to decidualization. The first-trimester decidual tissues do not exhibit intense stathmin expression. In other words, it is possible that the cells in these tissues have already been fully decidualized and thus no longer express stathmin. It is possible that the function played by stathmin in decidualization is no longer needed, once stromal cells have differentiated into decidual cells. This possibility is supported by our previous observation (Yoshie et al. 2004) that stathmin expression in rodent endometrial stromal cells decreases when these cells differentiate completely into decidual cells. These observations also support our hypothesis that stathmin participates in the preparation of decidualization.

The activity of stathmin is downregulated in response to a number of signals that elicit the post-transcriptional phosphorylation of four of its serine residues, Ser16, Ser25, Ser38, and Ser63.

Phosphorylation probably allows the creation of a local stathmin activity gradient, a process essential for regulating MT dynamics and spindle formation. In particular, the phosphorylation of Ser16 and Ser63 markedly downregulates the MT destabilizing activity of stathmin. The level of phosphorylated stathmin increases as cells enter the G2/M phases of the cell cycle and phosphorylation of stathmin on multiple sites is required for orderly progression through the cell cycle. The unphosphorylated form of stathmin promotes depolymerization of MT and phosphorylation of stathmin eliminates the ability of MT to depolymerize, thereby allowing the mitotic spindle to form. As both phosphorylation (inactivation) and the dephosphorylation (reactivation) of stathmin for cytokinesis are essential for the progression of cell cycle (Rubin \& Atweh 2004), the regulation of the cell cycle seems to be closely related to decidualization. In the present study, phosphorylated form of stathmin decreased acutely, whereas total stathmin levels decreased slowly during decidualization. The phosphorylation-dependent activity of stathmin might play an important role in the 
control of the dynamic instability of MTs during the decidualization of endometrial stromal cells. Increase in the relative ratio of active stathmin (non-phosphorylated form), which is caused by the enhancement of dephosphorylation might results in the marked reduction of phosphorylated stathmin levels. Changes in the ratio of non-phosphorylated and phosphorylated forms of stathmin may be important for decidual cell differentiation. Therefore, both total and phosphorylated stathmin levels in stromal cells decrease during decidualization is consistent with a previously published study that shows stathmin is highly expressed in undifferentiated cells, but decreases during the induction of cell differentiation (Schubart et al. 1992). Concerning the regulation of the cell cycle by stathmin, the stathmin promoter, which contains three E2F-binding sites, is regulated by E2F (Ishida et al. 2001), whose interaction with non-phosphorylated RB protein activity inhibits its activity (Bartek et al. 1996). On the other hand, the main function of cyclin $\mathrm{D}$ is thought to promote transcriptional regulation of $\mathrm{E} 2 \mathrm{~F}$ by releasing active $\mathrm{E} 2 \mathrm{~F}$ from the inactive form of complex of RB (Bartek et al. 1996). Further, cyclin-dependent kinase (CDK) inhibitor p2 $7^{\text {kip } 1}$ protein inhibits cell motility through impairment of the function of stathmin (Baldassarre et al. 2005, Rubin \& Atweh 2005). Therefore, stathmin expression might be regulated by cyclin D3.

In rodents, stromal cells show large mono- or bi-nucleated cells consisting of DNA with multiples of the haploid complement during decidualization. The expression of cyclin D3 during stromal cell decidualization has been shown to be related with polyploidization caused by an unusual cell cycle called endocycle. During an endocycle (endomitosis), nuclear replication occurs repeatedly without cell division. The coordinate expression and functional interaction of cyclin D3 with CDK4 are important for stromal cell proliferation, and the interaction of cyclin D3 with p21, an inhibitor of CDK, and CDK6 is important for development of polyploidy during decidualization (Tan et al. 2002). In mammals, megakaryocytes, a blood cell-type specialized to produce platelets, enter this unusual cell cycle during differentiation and become polyploidy (Ravid et al. 2002). Stathmin may be involved in this megakaryocyte polyploidization as well (Rubin et al. 2003). Therefore, the regulation of cell cycle, including polyploidization caused by stathmin seems to be important for the successful decidualization. Although we do not know whether the polyploidy occurs in human stromal cells, there is a possibility that stathmin expression is essential for the regulation of stromal cell cycle in decidual cells. The continuous expression of stathmin before decidualization and its downregulation in decidual cells might promote stromal cell differentiation. We speculate that the blockage of differentiation through impairment of the control of cell cycle results in the inhibition of IGFBP-1 and PRL.
Paclitaxel, an MT-stabilizing drugs, induces marked morphologic effect with mitotic arrest in the endometrium (Irving et al. 2000). We have recently observed that the treatment with vinblastine or colchicines (MT disrupting drugs) at the concentration between 1 and $100 \mathrm{pM}$ decreased the cAMP-induced IGFBP-1 secretion without changes in cell viability (data not shown). These results imply that the excess promotion of the MT destabilization diminishes the progression of decidualization, and the regulation of MT is associated with stromal cell differentiation. Accordingly, stathmin might play a significant role in morphological changes in stromal cells as well as differentiation.

In the placental tissues, we found stathmin is expressed in the cytotrophoblasts and extravillous trophoblasts, but not in the syncytiotrophoblasts. The chorionic villus is mainly comprised cytotrophoblasts and syncytiotrophoblasts. The syncytiotrophoblasts, which have multiple nuclei and secrete the human chorionic gonadotropin (hCG) and steroids required for pregnancy maintenance, are formed by the fusion and differentiation of cytotrophoblasts, which have single nuclei. Villous cytotrophoblasts also transform into extravillous trophoblasts and invade into the myometrium during the formation of decidua. It is possible that stathmin may play a role in cytotrophoblast differentiation and trophoblast invasion. Supporting this notion, a recent expression profiling study revealed that stathmin may be associated with trophoblastic differentiation (Rao et al. 2005). Thus, our observation of stathmin expression in placental villi suggests that stathmin may also be involved in the progressive changes in the placenta that take place during pregnancy.

\section{Acknowledgements}

The authors gratefully acknowledge Dr Andre Sobel (INSERM U440, Institut du Fer a Moulin, Paris, France) and Dr Parlow (Pituitary Hormone and Antisera Center) for providing the antistathmin antiserum and anti-human PRL antibody respectively. We also thank Dr Kazuhiko Imakawa at the University of Tokyo (Laboratory of Animal Breeding) for critically reading the manuscript. This work was partially supported by a Grantin-Aid for Scientific Research (16591689) to K T from the Japan Society for the Promotion of Science The authors declare that there is no conflict of interest that would prejudice the impartiality of this scientific work.

\section{References}

Baldassarre G, Belletti B, Nicoloso MS, Schiappacassi M, Vecchione A, Spessotto P, Morrione A, Canzonieri V \& Colombatti A 2005 p27(Kip1)-stathmin interaction influences sarcoma cell migration and invasion. Cancer Cell 7 51-63.

Bartek J, Bartkova J \& Lukas J 1996 The retinoblastoma protein pathway and the restriction point. Current Opinion in Cell Biology $\mathbf{8}$ 805-814.

Belmont LD \& Mitchison TJ 1996 Identification of a protein that interacts with tubulin dimers and increases the catastrophe rate of microtubules. Cell 84 623-631. 
Bieche I, Maucuer A, Laurendeau I, Lachkar S, Spano AJ, Frankfurter A, Levy P, Manceau V, Sobel A, Vidaud M, et al. 2003 Expression of stathmin family genes in human tissues: non-neural-restricted expression for SCLIP. Genomics 81 400-410.

Brosens JJ, Takeda S, Acevedo CH, Lewis MP, Kirby PL, Symes EK, Krausz T, Purohit A, Gellersen B \& White JO 2000 Human endometrial fibroblasts immortalized by simian virus 40 large $T$ antigen differentiate in response to a decidualization stimulus. Endocrinology 137 2225-2231.

Budwit-Novotny DA, McCarty KS, Cox EB, Soper JT, Mutch DG, Creasman WT, Flowers JL \& McCarty KS Jr 1986 Immunohistochemical analyses of estrogen receptor in endometrial adenocarcinoma using a monoclonal antibody. Cancer Research 46 5419-5425.

Curmi PA, Gavet O, Charbaut E, Ozon S, Lachkar-Colmerauer S, Manceau V, Siavoshian S, Maucuer A \& Sobel A 1999 Stathmin and its phosphoprotein family: general properties, biochemical and functional interaction with tubulin. Cell Structure and Function 24 345-357.

Di Paolo G, Pellier V, Catsicas M, Antonsson B, Catsicas S \& Grenningloh G 1996 The phosphoprotein stathmin is essential for nerve growth factor-stimulated differentiation. Journal of Cell Biology 133 1383-1390.

Drouva SV, Poulin B, Manceau V \& Sobel A 1998 Luteinizing hormonereleasing hormone-signal transduction and stathmin phosphorylation in the gonadotrope alphaT3-1 cell line. Endocrinology 139 2235-2239.

Gavet O, Ozon S, Manceau V, Lawler S, Curmi P \& Sobel A 1998 The stathmin phosphoprotein family: intracellular localization and effects on the microtubule network. Journal of Cell Science 111 3333-3346.

Gradin HM, Larsson N, Marklund U \& Gullberg M 1998 Regulation of microtubule dynamics by extracellular signals: cAMP-dependent protein kinase switches off the activity of oncoprotein 18 in intact cells. Journal of Cell Biology 140 131-141.

Hailat N, Strahler JR, Melhem R, Zhu XX, Brodeur G, Seeger RC, Reynolds CP \& Hanash S $1990 \mathrm{~N}$-myc gene amplification in neuroblastoma is associated with altered phosphorylation of a proliferation related polypeptide (Op 18). Oncogene 5 1615-1618.

Irving JA, McFarland DF, Stuart DS \& Gilks CB 2000 Mitotic arrest of endometrial epithelium after paclitaxel therapy for breast cancer. International Journal of Gynecological Pathology 19 395-397.

Irwin JC, Kirk D, King RJ, Quigley MM \& Gwatkin RB 1989 Hormonal regulation of human endometrial stromal cells in culture: an in vitro model for decidualization. Fertility and Sterility 52 761-768.

Ishida S, Huang E, Zuzan H, Spang R, Leone G, West M \& Nevins JR 2001 Role for E2F in control of both DNA replication and mitotic functions as revealed from DNA microarray analysis. Molecular and Cellular Biology 21 4684-4699.

Kasahara K, Takakura K, Takebayashi K, Kimura F, Nakanishi K \& Noda Y 2001 The role of human chorionic gonadotropin on decidualization of endometrial stromal cells in vitro. Journal of Clinical Endocrinology and Metabolism 86 1281-1286.

Nakashima D, Uzawa K, Kasamatsu A, Koike H, Endo Y, Saito K, Hashitani S, Numata T, Urade M \& Tanzawa H 2006 Protein expression profiling identifies maspin and stathmin as potential biomarkers of adenoid cystic carcinoma of the salivary glands. International Journal of Cancer 118 704-713.

Nogales E, Wang HW \& Niederstrasser H 2003 Tubulin rings: which way do they curve? Current Opinion in Structural Biology 13 256-261.

Noyes RW, Hertig AT \& Rock J 1950 Dating the endometrial biopsy. Fertility and Sterility 1 3-25.

Ozon S, Maucuer A \& Sobel A 1997 The stathmin family - molecular and biological characterization of novel mammalian proteins expressed in the nervous system. European Journal of Biochemistry 248 794-806.

Pasmantier R, Danoff A, Fleischer N \& Schubart UK 1986 P19, a hormonally regulated phosphoprotein of peptide hormone-producing cells: secretagogue-induced phosphorylation in AtT-20 mouse pituitary tumor cells and in rat and hamster insulinoma cells. Endocrinology 119 1229-1238.
Popovici RM, Kao LC \& Giudice LC 2000 Discovery of new inducible genes in in vitro decidualized human endometrial stromal cells using microarray technology. Endocrinology 141 3510-3513.

Price DK, Ball JR, Bahrani-Mostafavi Z, Vachris JC, Kaufman JS, Naumann RW, Higgins RV \& Hall JB 2000 The phosphoprotein Op18/stathmin is differentially expressed in ovarian cancer. Cancer Investigation 18 722-730.

Puissant C, Mitev V, Lemnaouar M, Manceau V, Sobel A \& Houdebine LM 1995 Stathmin gene expression in mammary gland and in Nb2 cells. Biology of the Cell 85 109-115.

Rao AJ, Neelima PS, Yashwanth R, Anbalgan M, Mahesh K, Dahia CL \& Rao RM 2005 Expression profiling of genes in BeWo cells following forskolin induced differentiation. Program of the 38th Annual Meeting of The Society for The Study of Reproduction, Quebec, Canada. pp 212.

Ravid K, Lu J, Zimmet JM \& Jones MR 2002 Roads to polyploidy: the megakaryocyte example. Journal of Cellular Physiology 190 7-20.

Rowlands DC, Williams A, Jones NA, Guest SS, Reynolds GM, Barber PC \& Brown G 1995 Stathmin expression is a feature of proliferating cells of most, if not all, cell lineages. Laboratory Investigation 72 100-113.

Rubin Cl, French DL \& Atweh GF 2003 Stathmin expression and megakaryocyte differentiation: a potential role in polyploidy. Experimental Hematology 31 389-397.

Rubin CI \& Atweh GF 2004 The role of stathmin in the regulation of the cell cycle. Journal of Cellular Biochemistry 93 242-250.

Rubin CI \& Atweh GF 2005 p $27^{\text {Kip } 1}$ and stathmin share the stage for the first time. Trends in Cell Biology 15 346-348.

Satyaswaroop PG, Bressler RS, de la Pena MM \& Gurpide E 1979 Isolation and culture of human endometrial glands. Journal of Clinical Endocrinology and Metabolism 48 639-641.

Schubart UK, Xu J, Fan W, Cheng G, Goldstein H, Alpini G, Shafritz DA, Amat JA, Farooq M, Norton WT, et al. 1992 Widespread differentiation stage-specific expression of the gene encoding phosphoprotein p19 (metablastin) in mammalian cells. Differentiation 51 21-32.

Tabanelli S, Tsang B \& Gurpide E 1992 In vitro decidualization of human endometrial stromal cells. Journal of Steroid Biochemistry and Molecular Biology 42 337-344.

Takekoshi K, Nomura F, Isobe K, Motooka M, Nammoku T \& Nakai T 1998 Identification and initial characterization of stathmin by the differential display method in nerve growth factor-treated PC12 cells. European Journal 138 707-712.

Tamura K, Kawaguchi T, Hara T, Sakamoto T, Tohei A, Miyajima A, Tsutiya S \& Kogo H 2000 Interleukin-6 decreases estrogen production and messenger ribonucleic acid expression encoding aromatase during in vitro cytodifferentiation of rat granulosa cell. Molecular and Cellular Endocrinology 170 103-111.

Tamura K, Hara T, Yoshie M, Irie S, Sobel A \& Kogo H 2003 Enhanced expression of uterine stathmin during the process of implantation and decidualization in rats. Endocrinology 144 1464-1473.

Tan J, Raja S, Davis MK, Tawfik O, Dey SK \& Das SK 2002 Evidence for coordinated interaction of cyclin D3 with p21 and cdk6 in directing the development of uterine stromal cell decidualization and polyploidy during implantation. Mechanisms of Development 111 99-113.

Tang B, Guller S \& Gurpide E 1993 Cyclic adenosine 3' , 5' -monophosphate induces PRL expression in stromal cells isolated from human proliferative endometrium. Endocrinology 133 2197-2203.

Yoshie M, Tamura K \& Kogo H 2004 Differential localization of decidual stathmin during pregnancy in rats. Placenta 25 449-455.

Yoshie M, Tamura K, Hara T \& Kogo H 2006 Expression of the stathmin family genes in the uterus during early pregnancy of mouse and their functions. Molecular Reproduction and Development 73 164-172.

Received 17 February 2006

First decision 26 April 2006

Revised manuscript received 17 June 2006

Accepted 14 July 2006 